\title{
The Optimal Upper and Lower Power Mean Bounds for a Convex Combination of the Arithmetic and Logarithmic Means
}

\author{
Wei-Feng Xia, ${ }^{1}$ Yu-Ming Chu, ${ }^{2}$ and Gen-Di Wang ${ }^{2}$ \\ ${ }^{1}$ School of Teacher Education, Huzhou Teachers College, Huzhou, Zhejiang 313000, China \\ ${ }^{2}$ Department of Mathematics, Huzhou Teachers College, Huzhou, Zhejiang 313000, China \\ Correspondence should be addressed to Yu-Ming Chu, chuyuming2005@yahoo.com.cn \\ Received 16 December 2009; Accepted 12 March 2010 \\ Academic Editor: Lance Littlejohn
}

Copyright (C) 2010 Wei-Feng Xia et al. This is an open access article distributed under the Creative Commons Attribution License, which permits unrestricted use, distribution, and reproduction in any medium, provided the original work is properly cited.

For $p \in \mathbb{R}$, the power mean $M_{p}(a, b)$ of order $p$, logarithmic mean $L(a, b)$, and arithmetic mean $A(a, b)$ of two positive real values $a$ and $b$ are defined by $M_{p}(a, b)=\left(\left(a^{p}+b^{p}\right) / 2\right)^{1 / p}$, for $p \neq 0$ and $M_{p}(a, b)=\sqrt{a b}$, for $p=0, L(a, b)=(b-a) /(\log b-\log a)$, for $a \neq b$ and $L(a, b)=a$, for $a=b$ and $A(a, b)=(a+b) / 2$, respectively. In this paper, we answer the question: for $\alpha \in(0,1)$, what are the greatest value $p$ and the least value $q$, such that the double inequality $M_{p}(a, b) \leq$ $\alpha A(a, b)+(1-\alpha) L(a, b) \leq M_{q}(a, b)$ holds for all $a, b>0$ ?

\section{Introduction}

For $p \in \mathbb{R}$, the power mean $M_{p}(a, b)$ of order $p$ and logarithmic mean $L(a, b)$ of two positive real values $a$ and $b$ are defined by

$$
\begin{array}{r}
M_{p}(a, b)= \begin{cases}\left(\frac{a^{p}+b^{p}}{2}\right)^{1 / p}, & p \neq 0, \\
\sqrt{a b}, & p=0,\end{cases} \\
L(a, b)= \begin{cases}\frac{b-a}{\log b-\log a}, & a \neq b, \\
a, & a=b,\end{cases}
\end{array}
$$

respectively. In the recent past, both mean values have been the subject of intensive research. In particular, many remarkable inequalities for power mean or logarithmic mean can 
be found in the literature [1-15]. It might be surprising that the logarithmic mean has applications in physics, economics, and even in meteorology [16-18]. In [16] the authors study a variant of Jensen's functional equation involving $L$, which appears in a heat conduction problem. A representation of $L$ as an infinite product and an iterative algorithm for computing the logarithmic mean as the common limit of two sequences of special geometric and arithmetic means are given in [11]. In $[19,20]$ it is shown that $L$ can be expressed in terms of Gauss's hypergeometric function ${ }_{2} F_{1}$. And, in [20] the authors prove that the reciprocal of the logarithmic mean is strictly totally positive, that is, every $n \times n$ determinant with elements $1 / L\left(a_{i}, b_{i}\right)$, where $0<a_{1}<a_{2}<\cdots<a_{n}$ and $0<b_{1}<b_{2}<\cdots<b_{n}$, is positive for all $n \geq 1$.

Let $A(a, b)=(1 / 2)(a+b), G(a, b)=\sqrt{a b}$, and $H(a, b)=2 a b /(a+b)$ be the arithmetic, geometric, and harmonic means of two positive numbers $a$ and $b$, respectively. Then it is well known that

$$
\begin{aligned}
\min \{a, b\} & \leq H(a, b)=M_{-1}(a, b) \leq G(a, b)=M_{0}(a, b) \\
& \leq L(a, b) \leq A(a, b)=M_{1}(a, b) \leq \max \{a, b\},
\end{aligned}
$$

and all inequalities are strict for $a \neq b$.

In [21], Alzer and Janous established the following best possible inequality:

$$
M_{\log 2 / \log 3}(a, b) \leq \frac{2}{3} A(a, b)+\frac{1}{3} G(a, b) \leq M_{2 / 3}(a, b)
$$

for all $a, b>0$.

In $[11,13,22]$ the authors present bounds for $L$ in terms of $G$ and $A$

$$
G^{2 / 3}(a, b) A^{1 / 3}(a, b)<L(a, b)<\frac{2}{3} G(a, b)+\frac{1}{3} A(a, b)
$$

for all $a, b>0$ with $a \neq b$.

The following sharp bounds for $L$ in terms of power means are proved by Lin [12]

$$
M_{0}(a, b)<L(a, b)<M_{1 / 3}(a, b) .
$$

The main purpose of this paper is to answer the question: for $\alpha \in(0,1)$, what are the greatest value $p$ and the least value $q$, such that the double inequality $M_{p}(a, b) \leq \alpha A(a, b)+$ $(1-\alpha) L(a, b) \leq M_{q}(a, b)$ holds for all $a, b>0$ ?

\section{Lemmas}

In order to establish our results we need several lemmas, which we present in this section. 
Lemma 2.1. If $\alpha \in(0,1)$, then $(1+2 \alpha)(\log 2-\log \alpha)>3 \log 2$.

Proof. For $\alpha \in(0,1)$, let $f(\alpha)=(1+2 \alpha)(\log 2-\log \alpha)$, then simple computations lead to

$$
\begin{aligned}
& f^{\prime}(\alpha)=2(\log 2-1)-2 \log \alpha-\frac{1}{\alpha^{\prime}} \\
& f^{\prime \prime}(\alpha)=\frac{1}{\alpha^{2}}(1-2 \alpha) .
\end{aligned}
$$

From (2.2) we clearly see that $f^{\prime \prime}(\alpha)>0$ for $\alpha \in(0,1 / 2)$, and $f^{\prime \prime}(\alpha)<0$ for $\alpha \in(1 / 2,1)$. Then from (2.1) we get

$$
f^{\prime}(\alpha) \leq f^{\prime}\left(\frac{1}{2}\right)=4(\log 2-1)<0
$$

for $\alpha \in(0,1)$.

Therefore $f(\alpha)>f(1)=3 \log 2$ for $\alpha \in(0,1)$ follows from (2.3).

Lemma 2.2. Let $\alpha \in(0,1)$, if $p=\log 2 /(\log 2-\log \alpha)$, then

$$
-p^{3}+(4 \alpha-1) p^{2}-3 \alpha p+\alpha<0 .
$$

Proof. For $\alpha \in(0,1)$, let $t=-\log \alpha$, then $t \in(0,+\infty)$ and

$$
-p^{3}+(4 \alpha-1) p^{2}-3 \alpha p+\alpha=\frac{f(t)}{(t+\log 2)^{3} e^{t}}
$$

where $f(t)=(t+\log 2)^{3}-3 \log 2(t+\log 2)^{2}+(\log 2)^{2}(t+\log 2)\left(4-e^{t}\right)-(\log 2)^{3} e^{t}$.

To prove Lemma 2.2 we need only to prove that $f(t)<0$ for $t \in(0,+\infty)$. Elementary calculations yield that

$$
\begin{gathered}
f(0)=0, \\
f^{\prime}(t)=3(t+\log 2)^{2}-6 \log 2(t+\log 2)-(\log 2)^{2} t e^{t}-(1+2 \log 2)(\log 2)^{2} e^{t}+4(\log 2)^{2},
\end{gathered}
$$




$$
\begin{gathered}
f^{\prime}(0)=-2(\log 2)^{3}<0, \\
\lim _{t \rightarrow+\infty} f^{\prime}(t)=-\infty, \\
f^{\prime \prime}(t)=6 t-(\log 2)^{2} t e^{t}-2(\log 2)^{2}(1+\log 2) e^{t}, \\
f^{\prime \prime}(0)=-2(1+\log 2)(\log 2)^{2}<0, \\
\lim _{t \rightarrow+\infty} f^{\prime \prime}(t)=-\infty, \\
f^{\prime \prime \prime}(t)=6-(\log 2)^{2} t e^{t}-(\log 2)^{2}(3+2 \log 2) e^{t}, \\
f^{\prime \prime \prime}(0)=6-3(\log 2)^{2}-2(\log 2)^{3}>0, \\
\lim _{t \rightarrow+\infty} f^{\prime \prime \prime}(t)=-\infty, \\
f^{(4)}(t)=-(\log 2)^{2} t e^{t}-2(\log 2)^{2}(2+\log 2) e^{t}<0
\end{gathered}
$$

for $t \in(0,+\infty)$.

Making use of a computer and the mathematica software, from (2.10) we get

$$
\begin{aligned}
& f^{\prime \prime}(1.15)=0.01679 \cdots, \\
& f^{\prime \prime}(1.16)=-0.0077 \cdots
\end{aligned}
$$

From (2.14)-(2.16) we clearly see that there exists a unique $t_{0} \in(0,+\infty)$, such that $f^{\prime \prime \prime}(t)>0$ for $t \in\left[0, t_{0}\right)$ and $f^{\prime \prime \prime}(t)<0$ for $t \in\left(t_{0},+\infty\right)$. Hence we know that $f^{\prime \prime}(t)$ is strictly increasing in $\left[0, t_{0}\right]$ and strictly decreasing in $\left[t_{0},+\infty\right)$.

From $(2.11),(2.12),(2.17),(2.18)$ and the monotonicity of $f^{\prime \prime}(t)$ in $\left[0, t_{0}\right]$ and in $\left[t_{0},+\infty\right)$ we know that there exist exactly two numbers $t_{1}, t_{2} \in(0,+\infty)$ with $t_{1}<t_{2}$, such that $f^{\prime \prime}(t)<0$ for $t \in\left[0, t_{1}\right) \cup\left(t_{2},+\infty\right)$ and $f^{\prime \prime}(t)>0$ for $t \in\left(t_{1}, t_{2}\right)$, and $t_{2}$ satisfies

$$
1.15<t_{2}<1.16
$$

Hence, we know that $f^{\prime}(t)$ is strictly decreasing in $\left[0, t_{1}\right] \cup\left[t_{2},+\infty\right)$ and strictly increasing in $\left[t_{1}, t_{2}\right]$.

Making use of a computer and the mathematica software, from (2.7) and (2.19), we get

$$
\begin{aligned}
f^{\prime}\left(t_{2}\right)< & 3(1.16+\log 2)^{2}-6 \log 2(1.15+\log 2)-1.15 \times e^{1.15} \times(\log 2)^{2} \\
& -(1+2 \log 2) \times(\log 2)^{2} \times e^{1.15}+4(\log 2)^{2} \\
= & -0.807 \cdots<0 .
\end{aligned}
$$


Now, $(2.8),(2.9),(2.20)$ and the monotonicity of $f^{\prime}(t)$ in $\left[0, t_{1}\right] \cup\left[t_{2},+\infty\right)$ and in $\left[t_{1}, t_{2}\right]$ imply that

$$
f^{\prime}(t)<0
$$

for $t \in(0,+\infty)$.

Therefore, $f(t)<0$ for $t \in(0,+\infty)$ follows from (2.6) and (2.21).

Lemma 2.3. For $\alpha \in(0,1)$ and $g(t)=\alpha\left(t-t^{p}\right)(\log t)^{2}+2(1-\alpha)\left(t+t^{p}\right) \log t-2(1-\alpha)(t-1)\left(1+t^{p}\right)$, one has the following.

(1) If $p=\log 2 /(\log 2-\log \alpha)$, then there exists $\lambda \in(1,+\infty)$ such that $g(t)>0$ for $t \in(1, \lambda)$ and $g(t)<0$ for $t \in(\lambda,+\infty)$.

(2) If $p=(1+2 \alpha) / 3$, then $g(t)<0$ for $t \in(1,+\infty)$.

Proof. Let $g_{1}(t)=t^{1-p} g^{\prime}(t), g_{2}(t)=t^{p} g_{1}^{\prime}(t), g_{3}(t)=t g_{2}^{\prime}(t), g_{4}(t)=t^{2-p} g_{3}^{\prime}(t), g_{5}(t)=t g_{4}^{\prime}(t)$, and $p \in\{\log 2 /(\log 2-\log \alpha),(1+2 \alpha) / 3\}$, then simple computations lead to

$$
\begin{gathered}
g(1)=0 \\
\lim _{t \rightarrow+\infty} g(t)=-\infty, \\
g_{1}(t)=\alpha\left(t^{1-p}-p\right)(\log t)^{2}+2\left(t^{1-p}+p-\alpha p-\alpha\right) \log t+2(1-\alpha)(1+p)(1-t), \\
g_{1}(1)=0, \\
\lim _{t \rightarrow+\infty} g_{1}(t)=-\infty, \\
g_{2}(t)=\alpha(1-p)(\log t)^{2}+2\left(1+\alpha-p-\alpha p t^{p-1}\right) \log t+2(p-\alpha p-\alpha) t^{p-1} \\
-2(1-\alpha)(1+p) t^{p}+2, \quad g_{2}(1)=0, \\
\lim _{t \rightarrow+\infty} g_{2}(t)=-\infty, \\
g_{3}(t)=2 \alpha(1-p)\left(1+p t^{p-1}\right) \log t+2\left[(1-\alpha) p^{2}-(1+\alpha) p+\alpha\right] t^{p-1} \\
-2 p(1-\alpha)(1+p) t^{p}+2(1+\alpha-p), \\
g_{3}(1)=2(1+2 \alpha-3 p), \\
\lim _{t \rightarrow+\infty} g_{3}(t)=-\infty,
\end{gathered}
$$




$$
\begin{gathered}
g_{4}(t)=2 \alpha(1-p) t^{1-p}-2 \alpha p(1-p)^{2} \log t-2 p^{2}(1-\alpha)(1+p) t \\
+2(p-1)\left[(1-\alpha) p^{2}-(1+2 \alpha) p+\alpha\right] \\
g_{4}(1)=2 p(1+2 \alpha-3 p) \\
\lim _{t \rightarrow+\infty} g_{4}(t)=-\infty \\
g_{5}(t)=2 \alpha(1-p)^{2} t^{1-p}-2 p^{2}(1-\alpha)(1+p) t-2 \alpha p(1-p)^{2}, \\
g_{5}(1)=-2\left[p^{3}-(4 \alpha-1) p^{2}+3 \alpha p-\alpha\right] \\
g_{5}^{\prime}(t)=2 \alpha(1-p)^{3} t^{-p}-2 p^{2}(1-\alpha)(1+p) \\
g_{5}^{\prime}(1)=-2\left[p^{3}-(4 \alpha-1) p^{2}+3 \alpha p-\alpha\right]
\end{gathered}
$$

(1) If $p=\log 2 /(\log 2-\log \alpha)$, then from (2.31), (2.34), (2.37)-(2.39), and Lemmas 2.1-2.2 we clearly see that

$$
\begin{aligned}
& g_{3}(1)>0, \\
& g_{4}(1)>0, \\
& g_{5}(1)<0, \\
& g_{5}^{\prime}(1)<0,
\end{aligned}
$$

and $g_{5}^{\prime}(t)$ is strictly decreasing in $[1,+\infty)$.

From (2.43) and the monotonicity of $g_{5}^{\prime}(t)$ we know that $g_{5}(t)$ is strictly decreasing in $[1,+\infty)$

The monotonicity of $g_{5}(t)$ and (2.42) implies that $g_{5}(t)<0$ for $t \in[1,+\infty)$, then we conclude that $g_{4}(t)$ is strictly decreasing in $[1,+\infty)$.

From the monotonicity of $g_{4}(t)$ and (2.35) together with (2.41) we clearly see that there exists $t_{1} \in(1,+\infty)$, such that $g_{4}(t)>0$ for $t \in\left[1, t_{1}\right)$ and $g_{4}(t)<0$ for $t \in\left(t_{1},+\infty\right)$. Hence we know that $g_{3}(t)$ is strictly increasing in $\left[1, t_{1}\right]$ and strictly decreasing in $\left[t_{1},+\infty\right)$.

The monotonicity of $g_{3}(t)$ in $\left[1, t_{1}\right]$ and in $\left[t_{1},+\infty\right)$ together with (2.32) and (2.40) imply that there exists $t_{2} \in(1,+\infty)$, such that $g_{3}(t)>0$ for $t \in\left[1, t_{2}\right)$ and $g_{3}(t)<0$ for $t \in\left(t_{2},+\infty\right)$. Then we know that $g_{2}(t)$ is strictly increasing in $\left[1, t_{2}\right]$ and strictly decreasing in $\left[t_{2},+\infty\right)$.

From (2.28) and (2.29) together with the monotonicity of $g_{2}(t)$ in $\left[1, t_{2}\right]$ and in $\left[t_{2},+\infty\right)$ we clearly see that there exists $t_{3} \in(1,+\infty)$, such that $g_{2}(t)>0$ for $t \in\left[1, t_{3}\right)$ and $g_{2}(t)<0$ for $t \in\left(t_{3},+\infty\right)$. Hence we know that $g_{1}(t)$ is strictly increasing in $\left[1, t_{3}\right]$ and strictly decreasing in $\left[t_{3},+\infty\right)$.

Equations (2.25) and (2.26) together with the monotonicity of $g_{1}(t)$ in $\left[1, t_{3}\right]$ and in $\left[t_{3},+\infty\right)$ imply that there exists $t_{4} \in(1,+\infty)$, such that $g_{1}(t)>0$ for $t \in\left[1, t_{4}\right)$ and $g_{1}(t)<0$ for $t \in\left(t_{4},+\infty\right)$. Then we conclude that $g(t)$ is strictly increasing in $\left[1, t_{4}\right]$ and strictly decreasing in $\left[t_{4},+\infty\right)$. 
Now (2.22), (2.23) and the monotonicity of $g(t)$ in $\left[1, t_{4}\right]$ and in $\left[t_{4},+\infty\right)$ imply that there exists $\lambda \in(1,+\infty)$, such that $g(t)>0$ for $t \in[1, \lambda)$ and $g(t)<0$ for $t \in(\lambda,+\infty)$.

(2) If $p=(1+2 \alpha) / 3$, then (2.31), (2.34), and (2.37)-(2.39) lead to

$$
\begin{gathered}
g_{4}(1)=g_{3}(1)=0, \\
g_{5}^{\prime}(1)=g_{5}(1)=-\frac{1}{27}\left[8\left(1-\alpha^{3}\right)+12 \alpha\left(1-\alpha^{2}\right)+60 \alpha^{2}(1-\alpha)\right]<0,
\end{gathered}
$$

and $g_{5}^{\prime}(t)$ is strictly decreasing in $[1,+\infty)$.

Therefore, Lemma 2.3(2) follows from (2.22), (2.25), (2.28), (2.44), (2.45), and the monotonicity of $g_{5}^{\prime}(t)$.

\section{Main Result}

Theorem 3.1. For $\alpha \in(0,1)$, the double inequality $M_{\log 2 /(\log 2-\log \alpha)}(a, b) \leq \alpha A(a, b)+(1-$ $\alpha) L(a, b) \leq M_{(1+2 \alpha) / 3}(a, b)$ holds for all $a, b>0$, each inequality becomes an equality if and only if $a=b$, and the given parameters $\log 2 /(\log 2-\log \alpha)$ and $(1+2 \alpha) / 3$ in each inequality are best possible.

Proof. If $a=b$, then from (1.1) and (1.2) we clearly see that $M_{\log 2 /(\log 2-\log \alpha)}(a, b)=\alpha A(a, b)+$ $(1-\alpha) L(a, b)=M_{(1+2 \alpha) / 3}(a, b)=a$ for $\alpha \in(0,1)$. Next, we assume that $a \neq b$.

Firstly, we prove that $M_{\log 2 /(\log 2-\log \alpha)}(a, b)<\alpha A(a, b)+(1-\alpha) L(a, b)<M_{(1+2 \alpha) / 3}(a, b)$ for $a, b>0$ with $a \neq b$.

Without loss of generality, we assume that $a>b$. Let $t=a / b>1$ and $p \in\{\log 2 /(\log 2-$ $\log \alpha),(1+2 \alpha) / 3\}$, then (1.1) and (1.2) leads to

$$
\alpha A(a, b)+(1-\alpha) L(a, b)-M_{p}(a, b)=b\left[\frac{\alpha(t+1) \log t+2(1-\alpha)(t-1)}{2 \log t}-\left(\frac{t^{p}+1}{2}\right)^{1 / p}\right] .
$$

Let

$$
f(t)=\log \left[\frac{\alpha(t+1) \log t+2(1-\alpha)(t-1)}{2 \log t}\right]-\frac{1}{p} \log \left(\frac{t^{p}+1}{2}\right),
$$

then

$$
\begin{gathered}
\lim _{t \rightarrow 1} f(t)=0, \\
f^{\prime}(t)=\frac{g(t)}{t[\alpha(t+1) \log t+2(1-\alpha)(t-1)]\left(1+t^{p}\right) \log t^{\prime}}
\end{gathered}
$$

where $g(t)=\alpha\left(t-t^{p}\right)(\log t)^{2}+2(1-\alpha)\left(t+t^{p}\right) \log t-2(1-\alpha)(t-1)\left(1+t^{p}\right)$. 
If $p=\log 2 /(\log 2-\log \alpha)$, then it is not difficult to verify that

$$
\lim _{t \rightarrow+\infty} f(t)=0
$$

From (3.4) and Lemma 2.3(1) we know that there exists $\lambda \in(1,+\infty)$, such that $f(t)$ is strictly increasing in $[1, \lambda]$ and strictly decreasing in $[\lambda,+\infty)$. Then (3.3) and (3.5) together with the monotonicity of $f(t)$ in $[1, \lambda]$ and in $[\lambda,+\infty)$ imply that $f(t)>0$ for $t \in(1,+\infty)$, and from (3.1) and (3.2) we know that $\alpha A(a, b)+(1-\alpha) L(a, b)>M_{\log 2 /(\log 2-\log \alpha)}(a, b)$ for all $a, b>0$ with $a \neq b$.

If $p=(1+2 \alpha) / 3$, then from Lemma 2.3(2) and (3.1)-(3.4) we clearly see that $\alpha A(a, b)+$ $(1-\alpha) L(a, b)<M_{(1+2 \alpha) / 3}(a, b)$ for all $a, b>0$ with $a \neq b$.

Secondly, we prove that the parameters $\log 2 /(\log 2-\log \alpha)$ and $(1+2 \alpha) / 3$ cannot be improved in each inequality.

For any $\varepsilon>0$ and $x>1$, from (1.1) and (1.2) we get

$$
\begin{aligned}
\lim _{x \rightarrow+\infty} \frac{M_{\log 2 /(\log 2-\log \alpha)+\varepsilon}(1, x)}{\alpha A(1, x)+(1-\alpha) L(1, x)} & =\frac{2}{\alpha} \times\left(\frac{1}{2}\right)^{(\log 2-\log \alpha) /(\log 2+\varepsilon(\log 2-\log \alpha))} \\
& >\frac{2}{\alpha} \times\left(\frac{1}{2}\right)^{(\log 2-\log \alpha) / \log 2} \\
& =1
\end{aligned}
$$

Inequality (3.6) implies that for any $\varepsilon>0$ there exists $X=X(\varepsilon)>1$, such that $M_{\log 2 /(\log 2-\log \alpha)+\varepsilon}(1, x)>\alpha A(1, x)+(1-\alpha) L(1, x)$ for $x \in(X,+\infty)$. Hence the parameter $\log 2 /(\log 2-\log \alpha)$ cannot be improved in the left-side inequality.

Next for $0<\varepsilon<(1+2 \alpha) / 3$, let $0<x<1$, then (1.1) and (1.2) leads to

$$
\begin{aligned}
& {[\alpha A(1,1+x)+(1-\alpha) L(1,1+x)]^{(1+2 \alpha-3 \varepsilon) / 3}-\left[M_{(1+2 \alpha) / 3-\varepsilon}(1,1+x)\right]^{(1+2 \alpha-3 \varepsilon) / 3}} \\
& \quad=\left[\frac{(1-\alpha) x+\alpha(1+x / 2) \log (1+x)}{\log (1+x)}\right]^{(1+2 \alpha-3 \varepsilon) / 3}-\frac{1+(1+x)^{(1+2 \alpha-3 \varepsilon) / 3}}{2} \\
& \quad=\frac{f(x)}{[\log (1+x)]^{(1+2 \alpha-3 \varepsilon) / 3}}
\end{aligned}
$$

where $f(x)=[(1-\alpha) x+\alpha(1+\alpha / 2) \log (1+x)]^{(1+2 \alpha-3 \varepsilon) / 3}-\left(\left(1+(1+x)^{(1+2 \alpha-3 \varepsilon) / 3}\right) / 2\right)[\log (1+$ $x)]^{(1+2 \alpha-3 \varepsilon) / 3}$.

Let $x \rightarrow 0$, making use of the Taylor expansion we get

$$
f(x)=\frac{1}{24} \varepsilon(1+2 \alpha-3 \varepsilon) x^{(1+2 \alpha-3 \varepsilon) / 3}\left[x^{2}+o\left(x^{2}\right)\right] .
$$

Equations (3.7) and (3.8) imply that for any $0<\varepsilon<(1+2 \alpha) / 3$ there exists $0<\delta=$ $\delta(\varepsilon, \alpha)<1$, such that $\alpha A(1,1+x)+(1-\alpha) L(1,1+x)>M_{(1+2 \alpha) / 3-\varepsilon}(1,1+x)$ for $x \in(0, \delta)$. Hence the parameter $(1+2 \alpha) / 3$ cannot be improved in the right-side inequality. 


\section{Acknowledgment}

This research is supported by the Innovation Team Foundation (no. T200924) and NSF (no. Y200908671) of the Department of Education of Zhejiang Province, and NSF (nos. Y7080106, Y7080185) of Zhejiang Province.

\section{References}

[1] B. Y. Long and Y. M. Chu, "Optimal inequalities for generalized logarithmic, arithmetic, and geometric means," Journal of Inequalities and Applications, vol. 2010, Article ID 806825, 10 pages, 2010.

[2] B. Y. Long and Y. M. Chu, "Optimal power mean bounds for the weighted geometric mean of classical means," Journal of Inequalities and Applications, vol. 2010, Article ID 905679, 8 pages, 2010.

[3] Y. M. Chu and W. F. Xia, "Inequalities for generalized logarithmic means," Journal of Inequalities and Applications, vol. 2009, Article ID 763252, 7 pages, 2009.

[4] Y. M. Chu and W. F. Xia, "Two sharp inequalities for power mean, geometric mean, and harmonic mean," Journal of Inequalities and Applications, vol. 2009, Article ID 741923, 6 pages, 2009.

[5] M. Y. Shi, Y. M. Chu, and Y. P. Jiang, "Optimal inequalities among various means of two arguments," Abstract and Applied Analysis, vol. 2009, Article ID 694394, 10 pages, 2009.

[6] S. H. Wu, "Generalization and sharpness of the power means inequality and their applications," Journal of Mathematical Analysis and Applications, vol. 312, no. 2, pp. 637-652, 2005.

[7] J. E. Pečarić, "Generalization of the power means and their inequalities," Journal of Mathematical Analysis and Applications, vol. 161, no. 2, pp. 395-404, 1991.

[8] A. O. Pittenger, "Inequalities between arithmetic and logarithmic means," Publikacije Elektrotehničkog Fakulteta. Univerzitet u Beogradu. Serija Matematika I Fizika, no. 678-715, pp. 15-18, 1980.

[9] P. S. Bullen, D. S. Mitrinović, and P. M. Vasić, Means and Their Inequalities, vol. 31 of Mathematics and Its Applications (East European Series), D. Reidel, Dordrecht, The Netherlands, 1988.

[10] F. Burk, "The Geometric, logarithmic, and arithmetic mean inequality," The American Mathematical Monthly, vol. 94, no. 6, pp. 527-528, 1987.

[11] B. C. Carlson, “The logarithmic mean," The American Mathematical Monthly, vol. 79, pp. 615-618, 1972.

[12] T. P. Lin, "The power mean and the logarithmic mean," The American Mathematical Monthly, vol. 81, pp. 879-883, 1974.

[13] J. Sándor, “A note on some inequalities for means," Archiv der Mathematik, vol. 56, no. 5, pp. 471-473, 1991.

[14] K. B. Stolarsky, “Generalizations of the logarithmic mean," Mathematics Magazine, vol. 48, pp. 87-92, 1975.

[15] H. Alzer and S. L. Qiu, "Inequalities for means in two variables," Archiv der Mathematik, vol. 80, no. 2, pp. 201-215, 2003.

[16] P. Kahlig and J. Matkowski, "Functional equations involving the logarithmic mean," Zeitschrift für Angewandte Mathematik und Mechanik, vol. 76, no. 7, pp. 385-390, 1996.

[17] A. O. Pittenger, "The logarithmic mean in $n$ variables," The American Mathematical Monthly, vol. 92, no. 2, pp. 99-104, 1985.

[18] G. Pólya and G. Szegö, Isoperimetric Inequalities in Mathematical Physics, Annals of Mathematics Studies, no. 27, Princeton University Press, Princeton, NJ, USA, 1951.

[19] B. C. Carlson, "Algorithms involving arithmetic and geometric means," The American Mathematical Monthly, vol. 78, pp. 496-505, 1971.

[20] B. C. Carlson and J. L. Gustafson, "Total positivity of mean values and hypergeometric functions," SIAM Journal on Mathematical Analysis, vol. 14, no. 2, pp. 389-395, 1983.

[21] H. Alzer and W. Janous, "Solution of problem 8," Crux Mathematicorum, vol. 13, pp. 173-178, 1987.

[22] E. B. Leach and M. C. Sholander, "Extended mean values. II," Journal of Mathematical Analysis and Applications, vol. 92, no. 1, pp. 207-223, 1983. 


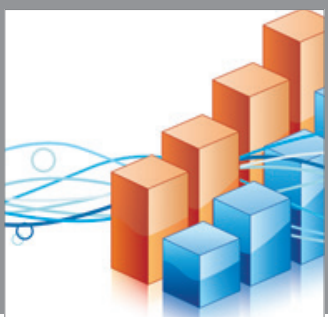

Advances in

Operations Research

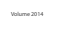

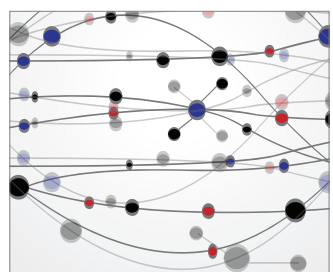

\section{The Scientific} World Journal
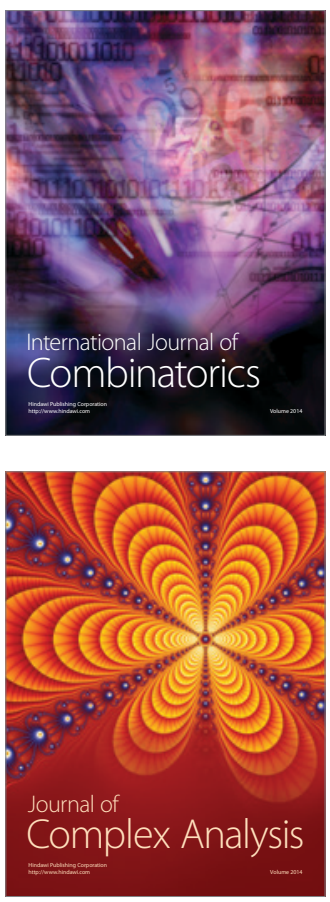

International Journal of

Mathematics and

Mathematical

Sciences
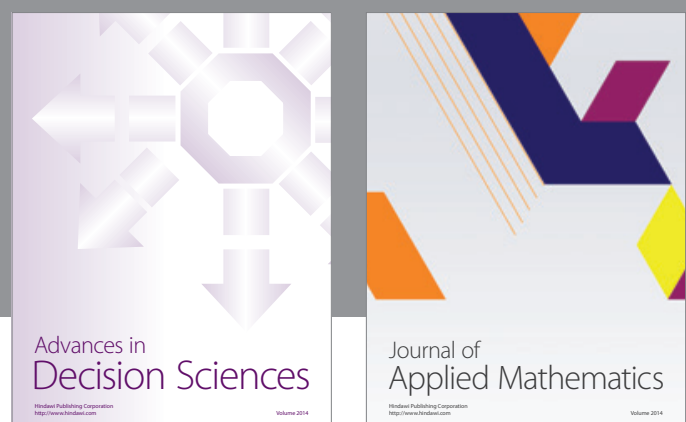

Journal of

Applied Mathematics
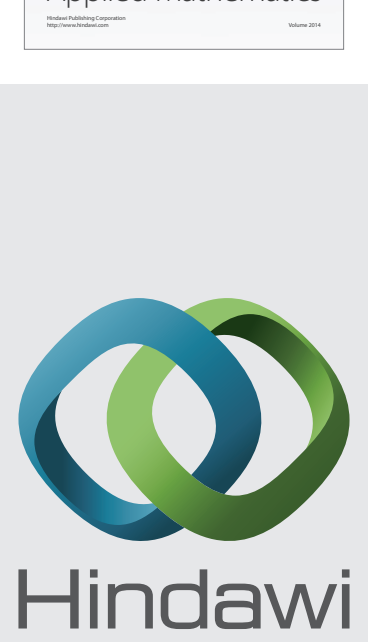

Submit your manuscripts at http://www.hindawi.com
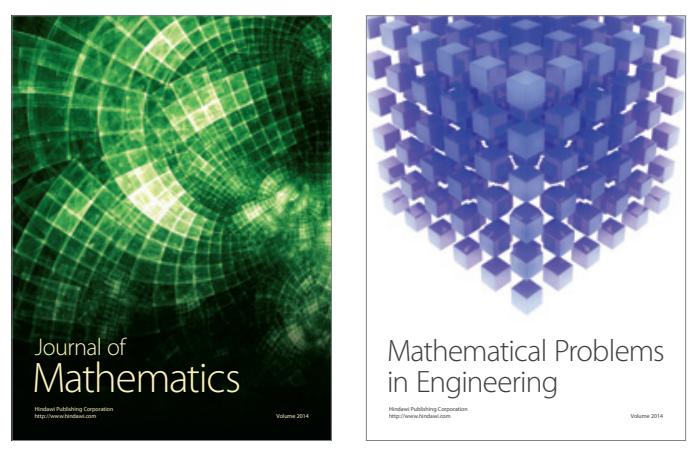

Mathematical Problems in Engineering
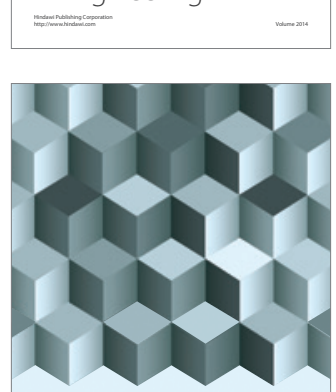

Journal of

Function Spaces
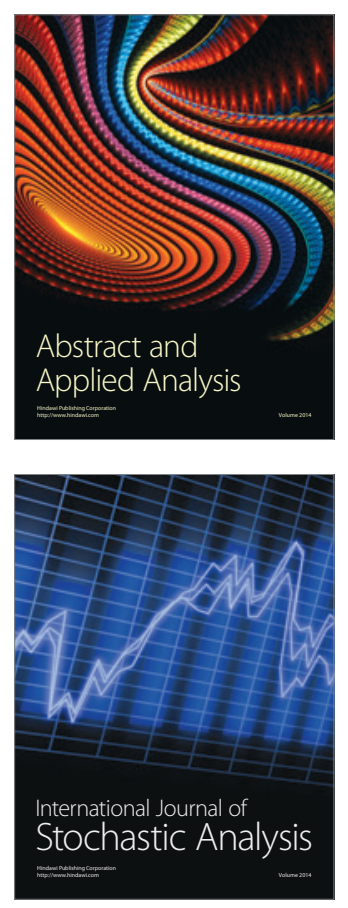

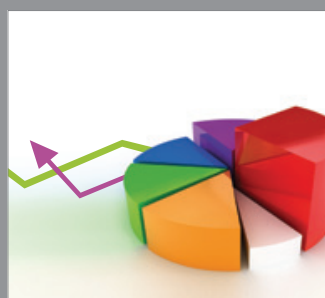

ournal of

Probability and Statistics

Promensencen
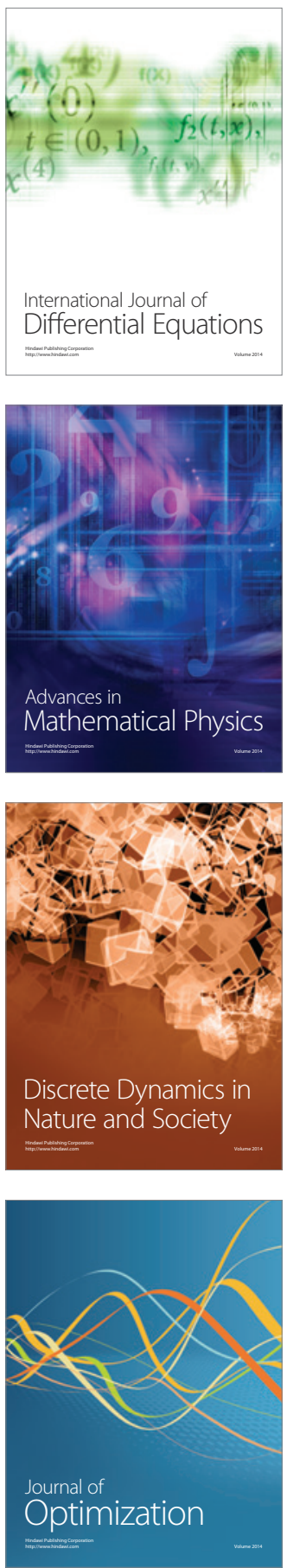